\title{
Phase diagram of diluted magnetic semiconductor quantum wells
}

\author{
L. Brey and F. Guinea \\ Instituto de Ciencia de Materiales (CSIC). Cantoblanco, 28049 Madrid. Spain.
}

(October 28, 2018)

\begin{abstract}
The phase diagram of diluted magnetic semiconductor quantum wells is investigated. The interaction between the carriers in the hole gas can lead to first order ferromagnetic transitions, which remain abrupt in applied fields. These transitions can be induced by magnetic fields or, in doublelayer systems by electric fields. We make a number of precise experimental predictions for observing these first order phase transitions.
\end{abstract}

Semiconductor and ferromagnetic materials have complementary properties in information processing and storage technologies. [1] Recent advances in the MBE growing techniques have made possible the fabrication of $M n$-based diluted magnetic semiconductors (DMS) with a rather high ferromagnetic-paramagnetic critical temperature $T_{c}$. [2] In semiconductors it is possible to modulate spatially the density of carriers by changing the doping profiles but in DMS it is also suitable to vary the magnetic order of the carriers by changing the magnetic ion densities. The combination of these two possibilities opens a rich field of applications for these materials.

The high critical temperature DMS's have a high concentration, $c$, of $\mathrm{Mn}^{2+}$ ions, randomly located. The itinerant carriers in the $G a_{1-x} M n_{x} A s$ systems are holes and their density, $c^{*}$, is much smaller than the magnetic ion density. In doped semiconductor the spin $S=5 / 2 \mathrm{Mn}^{2+}$ ions feel a long range ferromagnetic interaction created by the coupling mediated by the itinerant spin polarized carriers. [3 6] This interaction has some resemblance with the one observed in the pyrochlores [7, 8], where a similar type of coupling between a narrow electronic band and magnetic ions is assumed to exist. In the latter case, the phase diagram is significantly different from that of a conventional itinerant ferromagnet, showing first order transitions and phase separation [9] and/or the formation of localized textures near the transition temperature [10]. These features lead to interesting transport properties, like colossal magnetoresistance. We expect these effects to be amplified in low density two-dimensional (2D) doped semiconductors, where the carrier-carrier interaction can play a significant role, and also favors ferromagnetism [11].

In this work, we investigate the nature of the phase diagram of diluted magnetic semiconductor quantum wells, with emphasis on the existence of abrupt transitions for experimentally accessible parameters. We analyze quantum wells made of GaMnAs growth in the $<001>$ direction and with thickness $w$. The confinement of the carriers in the $z$-direction can be obtained by sandwiching the GaMnAs system between non-magnetic GaAlAs semiconductors.

The system is described by the following Hamiltonian,

$$
\mathcal{H}=\mathcal{H}_{M}+\mathcal{H}_{\text {holes }}+J \sum_{I} \mathbf{S}_{I} \cdot \mathbf{s}_{i} \delta\left(\mathbf{r}_{i}-\mathbf{R}_{I}\right)
$$

We now analyze the three terms contributing to $\mathcal{H}$. i) $\mathcal{H}_{M}$ is the Hamiltonian of the localized spins interacting with an external magnetic field $B$. Direct interactions between the magnetic moments of the $M n$ ions are much smaller than the interaction with the carrier spins and therefore, unlike in the case of the pyrochlores, we neglect this coupling.

ii) $\mathcal{H}_{\text {holes }}$ is the part of the Hamiltonian which describe the itinerant holes. It is the sum of the kinetic energy of the holes and the hole-hole interaction energy. We treat the kinetic energy of the carriers in the framework of the envelope function approximation. The confinement of the carriers in the quantum well produces the quantization of their motion and the appearance of subbands which, for sufficiently narrow quantum wells, have a clear light-hole or heavy-hole character. We assume that the hole density is low enough so that only one of the subbands, heavy-like, is occupied. With this, the in plane motion of the holes can be approximated by a single parabolic band of effective mass $m^{*}$. We describe the interaction between the electrical carriers with the local spin density approximation (LSDA) [12]. Except at very low densities, the LSDA gives an accurate description of the electron gas confined in quantum wells [16, 17]. Since the hole $g$-factor is much smaller than the $M n g$-factor we neglect in $\mathcal{H}_{\text {holes }}$ the coupling between the hole spins and the applied magnetic field.

iii)The last term is the antiferromagnetic exchange interaction between the spin of the $M n^{2+}$ ions located at $\mathbf{R}_{I}$ and the spins, $\overrightarrow{\mathbf{s}}_{i}$, of the itinerant carriers. The interaction between ions mediated by the conduction holes is of long range. Thus we will assume that thermal distribution of the orientation of the $M n$ spins is that induced by an effective field, due to the holes, which should be calculated selfconsistently.

In order to obtain the phase diagram of the DMS system we have to minimize the free energy $\mathcal{F}$. The critical temperatures for the ferromagnetic to paramagnetic transition in DMS is typically smaller than $100 K$, and for these temperatures we can consider that the electron gas is degenerate. Hence, the only temperature dependence in $\mathcal{F}$ is due to thermal fluctuations of the $M n$ spins. 
Treating the holes in the mean field approximation the free energy per unit area takes the form:

$$
\mathcal{F}=\mathcal{F}_{\text {ions }}+\frac{\hbar^{2}}{m^{*}} \frac{\pi}{2} n_{2 D}^{2}\left(1+\xi^{2}\right)+E_{x c}\left(n_{2 D}, \xi\right) .
$$

Here $\xi$ is the carrier spin polarization, $n_{2 D}=c^{*} w$ is the two dimensional density of holes in the system, $E_{x c}$ is the exchange correlation energy for the holes and $\mathcal{F}_{\text {ions }}$ is the contribution of the magnetic ions to the free energy:

$$
\mathcal{F}_{\text {ions }}=-T c w \log \frac{\sinh [\beta h(S+1 / 2)]}{\sinh (\beta h / 2)}
$$

being

$$
h=\frac{J}{2} \frac{n_{2 d}}{w} \xi+B
$$

the effective magnetic field than the $M n$ spins feel. In obtaining Eq. [3] we have assumed that the hole wave function in the $z$-direction has the form $w^{-1 / 2}$.

The phase diagram with parameters $J=0.15 \mathrm{eV} \mathrm{nm}^{3}$, ion concentration $c=1 \mathrm{~nm}^{-3}$ and width of the well, $d=$ $10 \mathrm{~nm}$, is shown in fig. $\{1$ 1 $\}$. We include a single hole band of effective mass $m_{\|}=0.11 m_{e}$, and a dielectric constant $\epsilon_{0}=12.2[6]$.

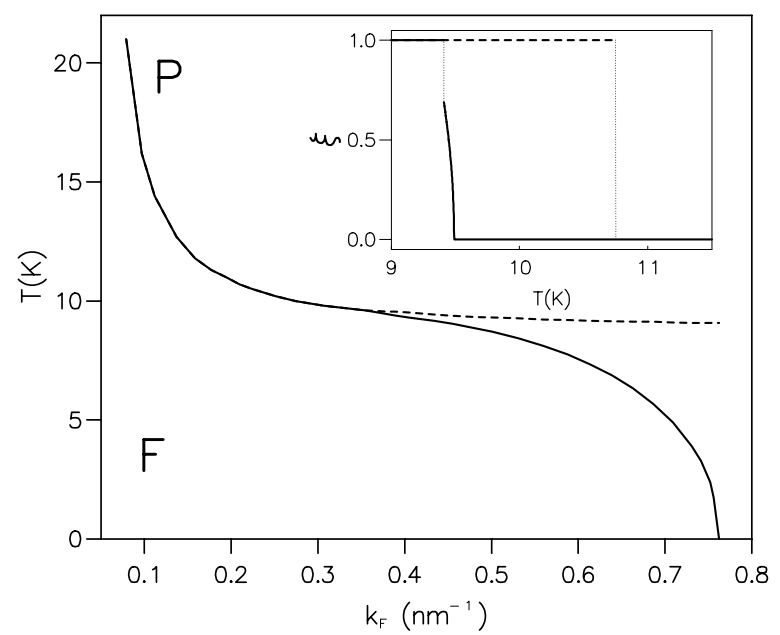

FIG. 1. Phase diagram of a magnetic semiconductor quantum well, using the parameters described in the text. Full line: first order transition. Broken line: second order phase transition. The holes in that phase to the left of the full line are completely polarized. The inset shows the polarization of the holes for $k_{F}=0.2 \mathrm{~nm}^{-1}$ (broken line) and $k_{F}=0.39 \mathrm{~nm}^{-1}$.

The calculations have been done by minimizing the value of the free energy, Eq. [2] and using the expression given by Vosko et al. for the exchange correlation potential. In the high density region the line of continuous transitions agrees with that obtained from the divergences of the magnetic susceptibility [6]. The main novelty of our calculation is the identification of a first order transition to a fully polarized state at intermediate densities. This transition takes place at higher temperatures than that at which the magnetic susceptibility of the system diverges. The existence of a first order transition between the paramagnetic and the ferromagnetic phases implies the existence of a discontinuity in the chemical potential at $T_{c}$. This discontinuity indicates the existence of a region in the phase diagram where phase separation can take place. At higher densities, when a continuous transition between $\xi=0$ and $\xi \neq 0$ occurs, we also find a first order phase transition between the partially polarized system, $\xi<1$, and the fully polarized phase, $\xi=1$.

In order to understand better the phase diagram it is illustrative to derive the existence of these first order transitions analytically. For doing that we treat the hole-hole interaction in the Hartree-Fock (HF) approximation. We obtain that the HF results are in rather good agreement with the obtained using the LSDA. In the HF approach the hole energy per unit area can be written as, 18

$$
\begin{aligned}
& E_{\text {holes }}=\frac{\hbar^{2}}{m^{*}} \frac{\pi}{2} n_{2 d}^{2}\left(1+\xi^{2}\right) \\
& -\frac{3 e^{2}}{8 \pi \epsilon_{0}}\left(\frac{3 \pi^{2}}{w}\right)^{1 / 3} n_{2 d}^{4 / 3}\left[(1+\xi)^{4 / 3}+(1-\xi)^{4 / 3}\right],
\end{aligned}
$$

where the last term is the exchange energy of the holes. The free energy of the ions is given by Eq. [3]. Near a paramagnetic-ferromagnetic transition, the hole spin polarization is small and we can expand the total free energy in terms of powers of $\xi$ :

$$
\begin{aligned}
\mathcal{F}_{t o t} \approx \frac{\hbar^{2}}{m^{*}} \frac{\pi}{2} n_{2 d}^{2}-C_{1} \frac{3}{4} \frac{n_{2 d}^{4 / 3}}{w^{1 / 3}}-T c w \log (2 S+1) \\
+\xi^{2}\left[\frac{\hbar^{2}}{m^{*}} \frac{\pi}{2} n_{2 d}^{2}-C_{1} \frac{1}{6} \frac{n_{2 d}^{4 / 3}}{w^{1 / 3}}-\frac{1}{T} \frac{c}{w} \frac{S(S+1)}{24} J^{2} n_{2 d}^{2}\right] \\
+\xi^{4}\left[-C_{1} \frac{5}{324} \frac{n_{2 d}^{4 / 3}}{w^{1 / 3}}+\frac{1}{T^{3}} \frac{c}{w^{3}} \frac{J^{4} n_{2 d}^{4}}{16} \times\right. \\
\left.\left(\frac{7}{360}\left(S+\frac{1}{2}\right)^{4}-\frac{S^{2}(S+1)^{2}}{72}\right)\right]+\ldots
\end{aligned}
$$

with

$$
C_{1}=\left(\frac{3}{\pi}\right)^{1 / 3} \frac{e^{2}}{\epsilon_{0}}
$$

We expect a paramagnetic-ferromagnetic transition when the quadratic term in $\xi$ is zero. This implies a critical temperature

$$
T_{C}=\frac{1}{12 w} \frac{c J^{2} S(S+1)}{\pi \frac{\hbar^{2}}{m^{*}}-\frac{1}{3 \pi} \frac{e^{2}}{\epsilon_{0}}\left(\frac{3 \pi^{2}}{w}\right)^{1 / 3} n_{2 d}^{-2 / 3}} .
$$

At high densities, this approximation gives $T_{C} \approx 8.7 \mathrm{~K}$, in good agreement with the LSDA calculation, and with 
the RKKY solution [6]. Note that the only dependence of $T_{c}$ on $n_{2 d}$ is through the hole-hole interaction. This is due to the fact that the two-dimensional density of states is energy independent. In three dimensional systems the kinetic energy scales as $n^{5 / 3}$ and $T_{c}$ is proportional to $n^{1 / 3}$ in agreement with the RKKY theory. The order of the transition can be inferred from the sign of the quartic term in Eq. [6]. If the quartic term is positive the transition is of second order, while a negative quartic term implies the existence of a first order phase transition. Using the previous expression for $T_{C}$, a first order transition takes place if:

$$
\begin{aligned}
\frac{e^{2}}{\epsilon_{0}} \frac{1}{\pi}\left(\frac{3 \pi^{2}}{w}\right)^{1 / 3} \frac{5}{2916} \frac{n_{2 d}^{-8 / 3}\left(\frac{7}{30}\left(S+\frac{1}{2}\right)^{4}-\frac{S^{2}(S+1)^{2}}{6}\right)}{c^{2} J^{2} S^{3}(S+1)^{3}} & \frac{1}{\left(\pi \frac{\hbar^{2}}{m^{*}}\right)^{3}\left(1-\frac{m^{*}}{\hbar^{2}} \frac{1}{3 \pi^{2}} \frac{e^{2}}{\epsilon_{0}}\left(\frac{3 \pi^{2}}{w}\right)^{1 / 3} n_{2 d}^{-2 / 3}\right)^{3}}>1
\end{aligned}
$$

which can be solved to give $n_{2 d}^{\text {first }} \approx 2.1 \times 10^{12} \mathrm{~cm}^{-2}$. This estimate is also in good agreement with the LSDA results shown in fig. $\{1]$.

Some comments about the LSDA exchange correlation potential are in order:

i)In the HF treatment, the existence of a negative quartic term in the expansion of the exchange energy in powers of $\xi$ is the source for the appearance of first order phase transitions. In the LSDA, the intermediate spin polarization correlation energy is obtained [13, 14, 12, 15] by assuming that it has the same polarization dependence as the exchange energy, so that the LSDA expression for $E_{x c}$ also leads to a negative quartic term when expanded in powers of $\xi$, and a first order transition is expected. On the other hand, correlation effects [14 weaken the spin dependence of the interaction energy as compared with the results in $\mathrm{HF}$, and we find that the LSDA gives a value for $n_{2 d}^{\text {first }}$ slightly smaller than the observed in the HF approximation. Numerical evaluation [14] of the partially spin polarized $E_{x c}$ also shows a negative quartic term in the expansion of $E_{x c}$ versus $\xi$. Hence, we believe that the existence of a first order phase transition is a robust result, independent of the model used for describing the interaction between carriers. Note, finally, that the existence of a first order transition implies the absence of long range critical fluctuations, lending further support to the adequacy of the methods used in this work.

ii)The interpolation formula used for describing the $\xi$ dependence of $E_{x c}$ is not analytic at $\xi=1$, Eq. [5]. This implies that there is, for each pair of values $n_{2 d}$ and $T$, a $\xi$ range near $\xi=1$ which cannot be reached by minimizing the total free energy. When $\partial \mathcal{F}^{2} / \partial \xi^{2}$ is smaller than zero, the system prefers to be completely spin polarized. More accurate descriptions of the LSDA $E_{x c}$ [14] results in an analytic behavior at $\xi=1$. Therefore we believe that, in the results shown in fig. $\{1]\}$, the discontinuous transition which occurs at lower temperatures than the second order transition $T_{c}$, is, probably, a spurious result due to the use of a HF-like interpolation for $E_{x c}$.

We remark again here that the appearance of a first order transition when decreasing $n_{2 d}$ is a real result, not related with the non analyticity of the LSDA expression for $E_{x c}$.

Now we analyze the effect in the phase diagram of an external magnetic field. For $B \neq 0$, the discontinuous transitions shown in fig. $\{1\}$ are changed into transitions between phases with two different polarizations. General thermodynamic arguments show that this line of first order transitions should end in a critical point, in an analogous way to the liquid-vapor phase diagram. This critical point belongs to the Ising universality class. In the present calculations, the first order transition persists at all fields. This is due to the non analytic of the exchange energy at $\xi=1$. We expect that a correct description of $E_{x c}$ would induce the termination of the line of first order transitions at a critical value of the field. Finite temperature effects in the hole gas, not taken into account, also will weaken the $\xi$ dependence of the free energy, leading to the existence of a critical point.

The line of discontinuous transitions in an applied field is shown in fig. $\{2\}$ \}.

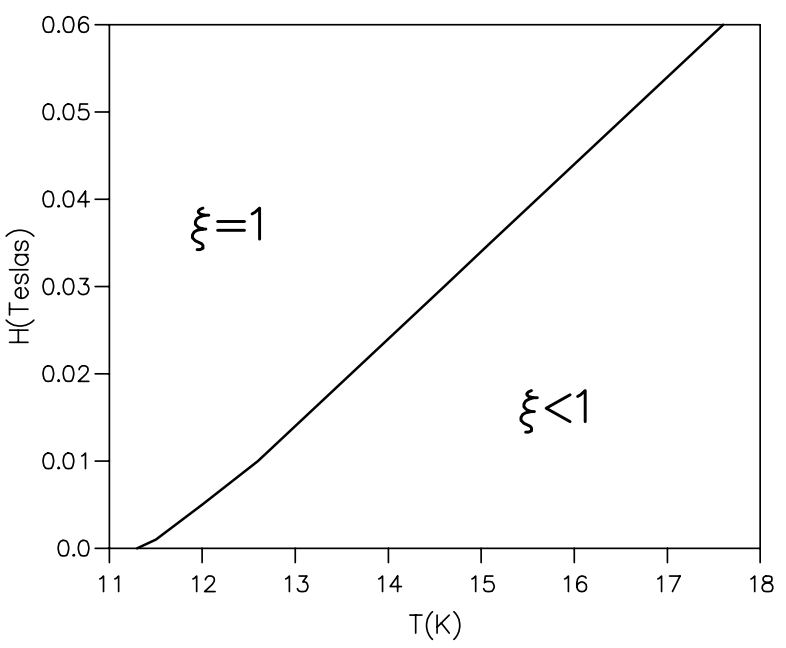

FIG. 2. Line of first order phase transitions as function of applied field and temperature, for fixed density.

The existence of discontinuous transitions leads to the possibility of phase separation. Using Maxwell construction, we find a region of phase separation near the line of first order transitions. In order to analyze its occurrence, we need to include electrostatic effects. The simplest situation where phase separation can be observed is in a bilayer. Let us imagine two wells with nominal hole density equal to $n_{0}$. At the temperatures and fields where the first order transition occurs, there are two phases with the same free energies, $\mathcal{F}_{1}\left(n_{0}\right)=\mathcal{F}_{2}\left(n_{0}\right)$. The chemical potential of these two phases differ at this point, and we define $\Delta \mu=\mu_{1}-\mu_{2}$. This difference in chemical potentials 
will induce a transfer of charge between the two layers, $\delta n$, until $\Delta \mu$ is compensated by the induced electrostatic potential, $V \approx\left(e^{2} \delta n d\right) / \epsilon_{0}$, where $d$ is the distance between the layers. Thus, we obtain $\delta n \approx\left(\Delta \mu \epsilon_{0}\right) /\left(e^{2} d\right)$. For reasonable values of $d \sim 10-50 \mathrm{~nm}$, we find that the charge transfer is small, $\delta n / n_{0} \sim 10^{-2}$. The change in the electrostatic barrier, $V \approx \Delta \mu$ is, however, comparable to the width of the band, and can change significantly the transport properties.

In a bilayer system, the first order transitions analyzed here can be induced by an applied electric field. The field induces a difference in the chemical potential of the two layers, which leads to a charge transfer. By suitably tuning the parameters, the density in one of the layers will reach the value at which the first order transition discussed above takes place. At this point, there will be an abrupt change in the charge transfer, which can be measured with standard capacitive techniques [16, 17]. The charge transfer as function of electric field, for reasonable parameters, is shown in fig. $\{[3\}$.

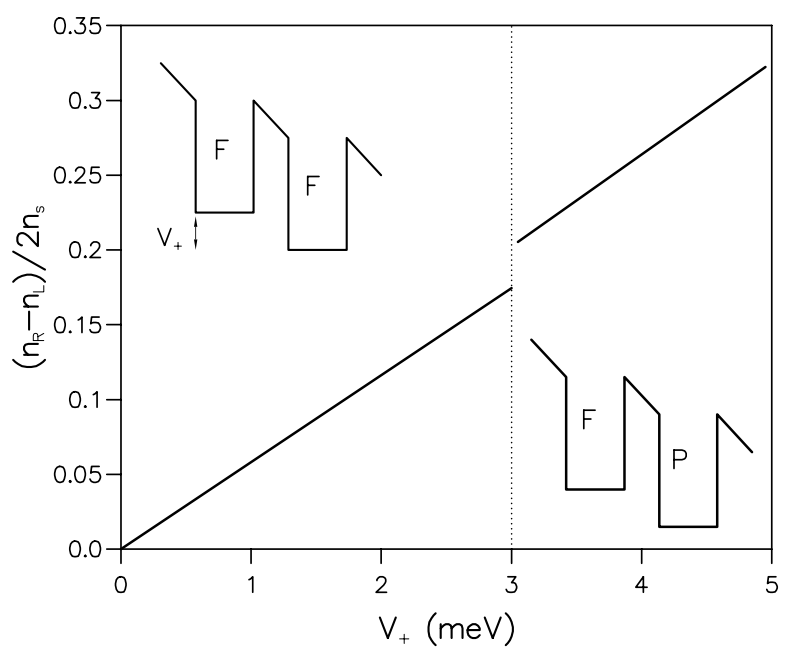

FIG. 3. Charge transfer in a bilayer system as function of the applied electric field.

In conclusion, we have analyzed the possible discontinuous transitions in two-dimensional diluted magnetic semiconductors. We find that the interaction between carriers can lead to first order transitions in twodimensional quantum wells. At the transition, the holes become fully polarized. This transition can be induced by a change in the density of the hole gas, the temperature, a magnetic field, and, in multilayer systems, an applied electric field. At these transitions, the minority spin band becomes empty, and the density of states at the Fermi level is reduced by one half. In double layer systems, significant electrostatic barriers can appear near the transition. This change can alter significantly the transport properties 20]. Thus, it can be important in the operation of devices made with these materials.
LB thanks A.H.MacDonald, C.Tejedor and A.Rubio for helpful discussions. We acknowledge financial support from grants PB96-0875 and PB96-0085 (MEC, Spain) and $(07 \mathrm{~N} / 0045 / 98$ and 07N/0027/99) (C. Madrid).

[1] See, e.g., G.A.Prinz, Science 282, 1660 (1998)

[2] H. Ohno, J. Magn. Magn. Mater. 200, 110 (1999); Science 281, 951 (1998).

[3] T.Dietl, A.Haury and Y.M.d'Aubigné, Phys.Rev.B 45, R3347 (1997).

[4] H.Takahashi, Phys.Rev.B, 56, 7839 (1997).

[5] T. Jungwirth, W. A. Atkinson, B. H. Lee and A. H. MacDonald, Phys. Rev. B 59, 9818 (1999).

[6] B. Lee, T. Jungwirth and A. H. MacDonald, preprint (cond-mat/0001391).

[7] Y. Shimikawa et al, Nature (London) 379, 53 (1996).

[8] J. A. Alonso et al, Phys. Rev. Lett. 82, 189 (1999).

[9] F. Guinea, G. Gómez-Santos and D. P. Arovas, Phys. Rev. B, in press.

[10] P. Majumdar and P. Littlewood, Phys. Rev. Lett. 81, 1314 (1998); M.J.Calderón, L.Brey and P.Littlewood cond-mat/9911109.

[11] L. Zheng, M. W. Ortalano and S. Das Sarma, Phys. Rev. B 55, 4506 (1997).

[12] S.H.Vosko, L.Wilk, M.Nusair Can.J.Phys. 58, 1200 (1980).

[13] U. von Barth and L. Hedin, J.Phys.C 5, 1629 (1972).

[14] O. Gunnarson and B. I. Lundqvist Phys. Rev. B 13, 4274 (1976).

[15] J. Perdew and A. Zunger, Phys. Rev. B 23, 5048 (1981).

[16] J. P. Eisenstein, L. N. Pfeiffer and K. W. West, Phys. Rev. Lett. 68, 674 (1992) and Phys. Rev. B 50, 1760 (1994).

[17] S. Ilani, A. Yacoby, D. Mahalu and H. Shtrikman, preprint (cond-mat/9910116).

[18] Many-Particle Theory. E.K.U. Gross, E.Runge and O.Heinonen, (Adan Hilger Ed.), Bristol (1991).

[19] T. Dietl, H. Ohno, F. Matsukura, J. Cibert and D. Ferrand, Science 287, 1019 (2000).

[20] J. C. Egues, Phys. Rev. Lett. 80, 4578 (1998). 